\author{
S. I. Fedynyak, P. V. Filevych
}

\title{
DISTANCE BETWEEN A MAXIMUM MODULUS POINT AND THE ZERO SET OF AN ANALYTIC FUNCTION
}

\begin{abstract}
S. I. Fedynyak, P. V. Filevych. Distance between a maximum modulus point and the zero set of an analytic function, Mat. Stud. 52 (2019), 10-23.

Let $f$ be an analytic function in the disk $\mathbb{D}_{R}=\{z \in \mathbb{C}:|z|<R\}, R \in(0,+\infty]$. A point $w \in \mathbb{D}_{R}$ is called a maximum modulus point of $f$ if $|f(w)|=M(|w|, f)$, where $M(r, f)=$ $\max \{|f(z)|:|z|=r\}$. Denote by $d(w, f)$ the distance between a maximum modulus point $w$ and the zero set of $f$, i.e., $d(w, f)=\inf \{|w-z|: f(z)=0\}$. Let $\Phi$ be a continuous function on $[a, \ln R)$ such that $x \sigma-\Phi(\sigma) \rightarrow-\infty, \sigma \uparrow \ln R$, for every $x \in \mathbb{R}$. Let also $\widetilde{\Phi}$ be the Youngconjugate function of $\Phi$ and $\bar{\Phi}(x)=\widetilde{\Phi}(x) / x$ for all sufficiently large $x$. We prove that if
\end{abstract}

$$
\ln M(r, f) \leq(1+o(1)) \Phi(\ln r), \quad r \uparrow R
$$

then

$$
\varliminf_{\mid \underline{w \mid \uparrow R}} d(w, f) \frac{\bar{\Phi}^{-1}(\ln |w|)}{|w|} \geq C_{0},
$$

where $C_{0}=0,5416 \ldots$ When the Taylor coefficients of $f$ are nonnegative, the constant $C_{0}$ can be replaced by $\pi$, and the inequality obtained in this case is sharp.

1. Introduction. Let $R \in(0,+\infty]$ and $\mathbb{D}_{R}=\{z \in \mathbb{C}:|z|<R\}$. By $\mathcal{A}_{R}$ denote the class of analytic functions in $\mathbb{D}_{R}$ of the form

$$
f(z)=\sum_{n=0}^{\infty} a_{n} z^{n}
$$

such that $f(z) \not \equiv 0$. For every function $f$ analytic in $\mathbb{D}_{R}$ put

$$
M(r, f)=\max \{|f(z)|:|z|=r\}, \quad r \in[0, R) .
$$

We call a point $w \in \mathbb{D}_{R}$ a maximum modulus point of $f$ if $|f(w)|=M(|w|, f)$.

Let $A \in(-\infty,+\infty]$ and $\Phi: D_{\Phi} \rightarrow \mathbb{R}$ be a real function. We say that $\Phi \in \Omega_{A}$ if the domain $D_{\Phi}$ of $\Phi$ is an infinite interval of the form $[a, A), \Phi$ is continuous on $D_{\Phi}$, and the following condition

$$
\forall x \in \mathbb{R}: \quad \lim _{\sigma \uparrow A}(x \sigma-\Phi(\sigma))=-\infty
$$

holds. It is easy to see that in the case $A<+\infty$ condition (2) is equivalent to the condition $\Phi(\sigma) \rightarrow+\infty, \sigma \rightarrow A-0$, and in the case $A=+\infty$ this condition is equivalent to the

2010 Mathematics Subject Classification:30B10, 30B30, 30C15, 30D20.

Keywords: analytic function; maximum modulus; maximum modulus point; zero set. doi:10.30970/ms.52.1.10-23

(C) S. I. Fedynyak, P. V. Filevych, 2019 
condition $\Phi(\sigma) / \sigma \rightarrow+\infty, \sigma \rightarrow+\infty$. For $\Phi \in \Omega_{A}$ by $\widetilde{\Phi}$ we denote the Young-conjugate function of $\Phi$, i.e.,

$$
\widetilde{\Phi}(x)=\max \left\{x \sigma-\Phi(\sigma): \sigma \in D_{\Phi}\right\}, \quad x \in \mathbb{R} .
$$

Note (see Lemma 2 below), that the function $\bar{\Phi}(x)=\widetilde{\Phi}(x) / x$ is continuous and increasing to $A$ on some interval of the form $\left(x_{0},+\infty\right)$. Hence the inverse function $\bar{\Phi}^{-1}$ is defined on some interval of the form $\left(A_{0}, A\right)$ and $\bar{\Phi}^{-1}$ is continuous and increasing to $+\infty$ on $\left(A_{0}, A\right)$.

For $f \in \mathcal{A}_{R}$ and $\Phi \in \Omega_{\ln R}$ the quantity

$$
T_{\Phi}(f)=\varlimsup_{r \uparrow R} \frac{\ln M(r, f)}{\Phi(\ln r)}
$$

is called $\Phi$-type of the function $f$. The notion of $\Phi$-type for a function analytic in $\mathbb{D}_{R}$ generalizes the classical notion of the type for an entire function of finite positive order.

Let $\mathcal{Z}_{R}$ be the class of functions $f \in \mathcal{A}_{R}$ that have infinitely many zeros in $\mathbb{D}_{R}$, and let $\mathcal{Z}_{R}^{+}$be the subclass of functions $f \in \mathcal{Z}_{R}$ of the form (1) such that $a_{n} \geq 0$ for all integers $n \geq 0$. Denote by $d(w, f)$ the distance between a maximum modulus point $w$ and the zero set of a function $f \in \mathcal{Z}_{R}$, that is

$$
d(w, f)=\inf \{|w-z|: f(z)=0\}
$$

The problem of finding asymptotic estimates from below for the quantity $d(w, f)$ as $|w| \rightarrow+\infty$ in the case where $f$ is entire was considered in the works of I. Ostrovskii [1], I. Ostrovskii and A.E. Üreyen [2, 3, 4], A.E. Üreyen [5], and S.I. Fedynyak [6]. In particular, by certain conditions on a function $\Phi \in \Omega_{+\infty}$, the authors of these works have specified a function $h:\left[r_{0},+\infty\right) \rightarrow(0,+\infty)$ dependent only on $\Phi$ such that the following statements are true.

$\left(O_{1}\right)$ If $f \in \mathcal{Z}_{+\infty}$ and $T_{\Phi}(f) \leq 1$, then

$$
\varliminf_{|w| \rightarrow+\infty} d(w, f) h(|w|) \geq \frac{1}{e}
$$

$\left(O_{2}\right)$ If $f \in \mathcal{Z}_{+\infty}^{+}$and $T_{\Phi}(f) \leq 1$, then

$$
\varliminf_{|w| \rightarrow+\infty} d(w, f) h(|w|) \geq 1
$$

$\left(O_{3}\right)$ There exists a function $f \in \mathcal{Z}_{+\infty}^{+}$such that $T_{\Phi}(f) \leq 1$ and

$$
\varliminf_{|w| \rightarrow+\infty} d(w, f) h(|w|) \leq \pi
$$

Next, we present an overview of relevant results. The first results in this direction, summarized in the following theorem, were obtained in [1].

Theorem A ([1]). Let $\rho$ and $T$ be positive numbers, $\Phi(\ln r)=\operatorname{Tr}^{\rho}$ and $h(r)=e T \rho r^{\rho-1}$ for all $r \geq r_{0}$. Then the statements $\left(O_{1}\right),\left(O_{2}\right)$, and $\left(O_{3}\right)$ are true.

A function $\rho(r)$, continuously differentiable on $\left[r_{0},+\infty\right)$, is called a proximate order if $\rho(r) \rightarrow \rho$ and $\rho^{\prime}(r) r \ln r \rightarrow 0$ as $r \rightarrow+\infty$ for some $\rho \in(0,+\infty)$. 
Theorem B ([2]). Let $\rho$ and $T$ be positive numbers, $\rho(r)$ be a proximate order such that $\rho(r) \rightarrow \rho$ as $r \rightarrow+\infty, \Phi(\ln r)=T r^{\rho(r)}$ and $h(r)=e T \rho r^{\rho(r)-1}$ for all $r \geq r_{0}$. Then the statements $\left(O_{1}\right),\left(O_{2}\right)$, and $\left(O_{3}\right)$ are true.

As in [5], a function $\rho(r)$, continuously differentiable on $\left[r_{0},+\infty\right)$, is called a zero proximate order if $\rho(r)=\psi(\ln r) / \ln r$ for all $r \geq r_{0}$, where $\psi$ is a function, positive concave on $\left[\ln r_{0},+\infty\right)$, and also $\rho(r) \rightarrow 0$ and $r^{\rho(r)} / \ln r \rightarrow+\infty$ as $r \rightarrow+\infty$.

Theorem $\mathbf{C}([5])$. Let $T$ be a positive number, $\rho(r)$ be a zero proximate order, $\Phi(\ln r)=$ $\operatorname{Tr}^{\rho(r)}$ and $h(r)=e \operatorname{Tr}^{\rho(r)-1}\left(\rho(r)+\rho^{\prime}(r) r \ln r\right)$ for all $r \geq r_{0}$. Then the statements $\left(O_{1}\right)$ and $\left(\mathrm{O}_{3}\right)$ are true.

As in [5], a function $\rho(r)$, continuously differentiable on $\left[r_{0},+\infty\right)$, is called an infinite proximate order if $\rho(r)=\psi(\ln r) / \ln r$ for all $r \geq r_{0}$, where $\psi$ is a function, positive increasing convex on $\left[\ln r_{0},+\infty\right.$ ), such that $\psi^{\prime}(x) \rightarrow+\infty$ and $\psi^{\prime \prime}(x) / \psi^{\prime 2}(x) \rightarrow 0$ (along the set of $x$ for which $\psi^{\prime \prime}(x)$ exists), as $x \rightarrow+\infty$.

Theorem D ([5]). Let $T$ be a positive number, $\rho(r)$ be a infinite proximate order, $\Phi(\ln r)=$ $\operatorname{Tr}^{\rho(r)}$ and $h(r)=e \operatorname{Tr}^{\rho(r)-1}\left(\rho(r)+\rho^{\prime}(r) r \ln r\right)$ for all $r \geq r_{0}$. Then the statements $\left(O_{1}\right)$ and $\left(\mathrm{O}_{3}\right)$ are true.

Finally, the following theorem is proved in [6].

Theorem $\mathbf{E}([6])$. Let $\Phi \in \Omega_{+\infty}$ be a function, continuously differentiable on $D_{\Phi}$, such that $\Phi^{\prime}$ is increasing on $D_{\Phi}$, and

$$
h(r)=\frac{1}{r} \cdot \bar{\Phi}^{-1}\left(\ln r+\frac{1}{\Phi^{-1}(\ln r)}\right)
$$

for all $r \geq r_{0}$. Then the statement $\left(O_{1}\right)$ is true.

Remark 1. It follows from Lemma 4 (see below) that for the function $h$ in Theorem $\mathrm{E}$ we have $h(r) \sim \bar{\Phi}^{-1}(\ln r) / r, r \rightarrow+\infty$.

Remark 2. For the function $h$ in Theorems A, B, C, and D, we have $h(r) \sim e \Phi^{\prime}(\ln r) / r$, $r \rightarrow+\infty$. Using this relation, we can prove that in each of the cases, considered in these theorems, the following inequality

$$
\varliminf_{r \rightarrow+\infty} \frac{r h(r)}{\bar{\Phi}^{-1}(\ln r)} \geq 1
$$

is true (we will not dwell on proving this fact).

Below we prove that the statements $\left(O_{1}\right),\left(O_{2}\right)$, and $\left(O_{3}\right)$ are true for an arbitrary function $\Phi \in \Omega_{+\infty}$ with $h(r)=\bar{\Phi}^{-1}(\ln r) / r, r \geq 0$. Moreover, the constant $\frac{1}{e}$ in (3) can be replaced by the larger constant

$$
C_{0}:=\max _{x>1} \frac{\ln (2 \sqrt{x(x-1)})}{x}=0,5416 \ldots
$$

and the constant 1 in (4) can be replaced by the sharp constant $\pi$.

At the same time, we prove similar results for functions analytic in a disk. 


\section{Main results.}

Theorem 1. Let $R \in(0,+\infty]$ and $\Phi \in \Omega_{\ln R}$. If $f \in \mathcal{Z}_{R}$ and $T_{\Phi}(f) \leq 1$, then

$$
\varliminf_{|w| \uparrow R} d(w, f) \frac{\bar{\Phi}^{-1}(\ln |w|)}{|w|} \geq C_{0} .
$$

Theorem 2. Let $R \in(0,+\infty]$ and $\Phi \in \Omega_{\ln R}$. If $f \in \mathcal{Z}_{R}^{+}$and $T_{\Phi}(f) \leq 1$, then

$$
\underset{|w| \uparrow R}{\lim } d(w, f) \frac{\bar{\Phi}^{-1}(\ln |w|)}{|w|} \geq \pi
$$

If $f \in \mathcal{A}_{R}$ is a function of the form (1), $\Phi \in \Omega_{\ln R}$, and $r \in[0, R)$, then let

$$
\mu(r, f)=\max \left\{\left|a_{n}\right| r^{n}: n \geq 0\right\}, \quad t_{\Phi}(f)=\varlimsup_{r \uparrow R} \frac{\ln \mu(r, f)}{\Phi(\ln r)} .
$$

Theorem 3. Let $R \in(0,+\infty]$ and $\Phi \in \Omega_{\ln R}$. Then there exists a function $f \in \mathcal{Z}_{R}^{+}$such that $T_{\Phi}(f)=t_{\Phi}(f)=1$ and

$$
\underset{|w| \uparrow R}{\lim } d(w, f) \frac{\bar{\Phi}^{-1}(\ln |w|)}{|w|}=\pi
$$

We need the following theorem to prove Theorem 2.

Theorem 4. Let $R \in(0,+\infty], f \in \mathcal{Z}_{R}^{+}$be a function of the form (1), and $\left(r_{j} e^{i \varphi_{j}}\right)$ be the sequence of all zeros of the function $f$. If $N:[0, R) \rightarrow(0,+\infty)$ is a function such that

$$
\sum_{n>N(r)} a_{n} r^{n}=o(M(r, f)), \quad r \uparrow R
$$

then

$$
\varliminf_{j \rightarrow \infty}\left|\varphi_{j}\right| N\left(r_{j}\right) \geq \pi
$$

\section{Auxiliary results.}

Lemma 1. Let $R \in(0,+\infty], \rho \in(0, R)$, and $f \in \mathcal{A}_{R}$. Then for every $w, z \in \mathbb{D}_{\rho}$ we have

$$
|f(w)-f(z)| \leq|w-z| \frac{\rho M(\rho, f)}{\sqrt{\left(\rho^{2}-|w|^{2}\right)\left(\rho^{2}-|z|^{2}\right)}} .
$$

Proof. We note that

$$
\int_{0}^{2 \pi} \frac{d x}{a-b \cos x}=\frac{2 \pi}{\sqrt{a^{2}-b^{2}}}
$$

whenever $a>b>0$. Hence, using Cauchy's integral formula and Cauchy-Bunyakovsky inequality, we obtain

$$
|f(w)-f(z)|=\left|\frac{1}{2 \pi i} \int_{|\tau|=\rho}\left(\frac{f(\tau)}{\tau-w}-\frac{f(\tau)}{\tau-z}\right) d \tau\right|=\frac{|w-z|}{2 \pi}\left|\int_{|\tau|=\rho} \frac{f(\tau)}{(\tau-w)(\tau-z)} d \tau\right|=
$$




$$
\begin{gathered}
=\frac{|w-z|}{2 \pi}\left|\int_{0}^{2 \pi} \frac{f\left(\rho e^{i \theta}\right)}{\left(\rho e^{i \theta}-w\right)\left(\rho e^{i \theta}-z\right)} \rho e^{i \theta} i d \theta\right| \leq \\
\leq \frac{|w-z|}{2 \pi} \rho M(\rho, f) \int_{0}^{2 \pi} \frac{d \theta}{\left|\rho e^{i \theta}-w\right|\left|\rho e^{i \theta}-z\right|} \leq \\
\leq \frac{|w-z|}{2 \pi} \rho M(\rho, f) \sqrt{\int_{0}^{2 \pi} \frac{d \theta}{\left|\rho e^{i \theta}-w\right|^{2}}} \sqrt{\int_{0}^{2 \pi} \frac{d \theta}{\left|\rho e^{i \theta}-z\right|^{2}}}= \\
=\frac{|w-z|}{2 \pi} \rho M(\rho, f) \sqrt{\frac{2 \pi}{\rho^{2}-|w|^{2}}} \sqrt{\frac{2 \pi}{\rho^{2}-|z|^{2}}}=|w-z| \frac{\rho M(\rho, f)}{\sqrt{\left(\rho^{2}-|w|^{2}\right)\left(\rho^{2}-|z|^{2}\right)}}
\end{gathered}
$$

The following lemma is well known (see, for example, [7, § 3.2], [8]).

Lemma 2. Let $A \in(-\infty,+\infty], \Phi \in \Omega_{A}$, and $\varphi(x)=\max \left\{\sigma \in D_{\Phi}: x \sigma-\Phi(\sigma)=\widetilde{\Phi}(x)\right\}$, $x \in \mathbb{R}$. Then, the following statements are true:

(i) the function $\varphi$ is nondecreasing on $\mathbb{R}$;

(ii) the function $\varphi$ is continuous from the right on $\mathbb{R}$;

(iii) $\varphi(x) \rightarrow A, x \rightarrow+\infty$;

(iv) the right-hand derivative of $\widetilde{\Phi}(x)$ is equal to $\varphi(x)$ at every point $x \in \mathbb{R}$;

(v) if $x_{0}=\inf \{x>0: \Phi(\varphi(x))>0\}$, then the function $\bar{\Phi}(x)=\widetilde{\Phi}(x) / x$ increases to $A$ on $\left(x_{0},+\infty\right)$

(vi) the function $\alpha(x)=\Phi(\varphi(x))$ is nondecreasing on $[0,+\infty)$.

In the following two lemmas, $\varphi$ and $x_{0}$ are defined by $\Phi$ in the same way as in Lemma 2 .

Lemma 3. Let $A \in(-\infty,+\infty], \Phi \in \Omega_{A}, \sigma_{0}=\bar{\Phi}\left(x_{0}+0\right)$, and $\sigma \in\left(\sigma_{0}, A\right)$. Then the minimum value of the function

$$
h(y)=\frac{\Phi(y)}{y-\sigma}, \quad y \in(\sigma, A),
$$

is $\bar{\Phi}^{-1}(\sigma)$, which is achieved when $y=\varphi\left(\bar{\Phi}^{-1}(\sigma)\right)$.

Proof. Let $x=\bar{\Phi}^{-1}(\sigma), y \in(\sigma, A)$. For $x>x_{0} \geq 0$ from the definition of function $\widetilde{\Phi}$ we obtain

$$
\sigma=\bar{\Phi}(x) \geq y-\frac{\Phi(y)}{x}=y-\frac{\Phi(y)}{\bar{\Phi}^{-1}(\sigma)},
$$

so that $\frac{\Phi(y)}{y-\sigma} \geq \bar{\Phi}^{-1}(\sigma)$. Thus $h(y) \geq \bar{\Phi}^{-1}(\sigma)$ for all $y \in(\sigma, A)$.

We note that by Lemma 2

$$
x \varphi(x)-\Phi(\varphi(x))=\widetilde{\Phi}(x), \quad x \in \mathbb{R} .
$$

If $x>x_{0}$, then $\Phi(\varphi(x))>0$, thus from (12) we obtain $\bar{\Phi}(x)<\varphi(x)<A$. For $x=\bar{\Phi}^{-1}(\sigma)$ and $y=\varphi\left(\bar{\Phi}^{-1}(\sigma)\right)=\varphi(x)$ we have $y \in(\sigma, A)$. Then, by (12),

$$
h(y)=\frac{\Phi(\varphi(x))}{\varphi(x)-\bar{\Phi}(x)}=x=\bar{\Phi}^{-1}(\sigma) .
$$


Lemma 4. Let $\delta \in(0,1), A \in(-\infty,+\infty], \Phi \in \Omega_{A}, \sigma_{0}=\bar{\Phi}\left(x_{0}+0\right)$, and $y(\sigma)=\varphi\left(\bar{\Phi}^{-1}(\sigma)\right)$ for all $\sigma \in\left(\sigma_{0}, A\right)$. Then

$$
\bar{\Phi}^{-1}\left(\sigma+\frac{\delta \Phi(y(\sigma))}{\bar{\Phi}^{-1}(\sigma)}\right) \leq \frac{\bar{\Phi}^{-1}(\sigma)}{1-\delta}, \quad \sigma \in\left(\sigma_{0}, A\right) .
$$

Proof. By Lemma 3 for all $\sigma \in\left(\sigma_{0}, A\right)$ we have

$$
\frac{\Phi(y(\sigma))}{y(\sigma)-\sigma}=\bar{\Phi}^{-1}(\sigma)
$$

Hence we get

$$
y(\sigma)=\sigma+\frac{\Phi(y(\sigma))}{\bar{\Phi}^{-1}(\sigma)}
$$

Put

$$
v(\sigma)=\sigma+\frac{\delta \Phi(y(\sigma))}{\bar{\Phi}^{-1}(\sigma)} .
$$

Clearly, if $\sigma \in\left(\sigma_{0}, A\right)$, then $\sigma<v(\sigma)<y(\sigma)$ and also $y(\sigma)-v(\sigma)=(1-\delta)(y(\sigma)-\sigma)$. Therefore, using Lemma 3 again, for every $\sigma \in\left(\sigma_{0}, A\right)$ we obtain

$$
\bar{\Phi}^{-1}(v(\sigma)) \leq \frac{\Phi(y(\sigma))}{y(\sigma)-v(\sigma)}=\frac{\Phi(y(\sigma))}{(1-\delta)(y(\sigma)-\sigma)}=\frac{\bar{\Phi}^{-1}(\sigma)}{1-\delta} .
$$

This proves Lemma 4.

Lemma 5. Let $A \in(-\infty,+\infty], \Phi \in \Omega_{A}$, and $\Psi$ be a convex function on $\left[\sigma_{1}, A\right)$ such that $\Psi(\sigma) \leq \Phi(\sigma)$ for all $\sigma \in\left[\sigma_{1}, A\right)$. Then $\Psi_{+}^{\prime}(\sigma) \leq \bar{\Phi}^{-1}(\sigma)$ for every $\sigma \in\left[\sigma_{2}, A\right)$.

Proof. Since $\Phi(\sigma) \rightarrow+\infty, \sigma \uparrow A$, from the conditions imposed on the function $\Psi$ we get $\Psi(y)-\Psi(\sigma) \leq \Phi(y)$ for all $\sigma \in\left[\sigma_{2}, A\right)$ and $y \in(\sigma, A)$. We can assume that $\sigma_{2} \geq \sigma_{1}$ and $\sigma_{2} \in\left(\sigma_{0}, A\right)$, where $\sigma_{0}$ is the constant from Lemma 3. Set $y=\varphi\left(\bar{\Phi}^{-1}(\sigma)\right)$. Since $\Psi$ is a convex function on $\left[\sigma_{1}, A\right)$, for every $\sigma \in\left[\sigma_{2}, A\right)$ we get, using Lemma 3,

$$
\Psi_{+}^{\prime}(\sigma) \leq \frac{\Psi(y)-\Psi(\sigma)}{y-\sigma} \leq \frac{\Phi(y)}{y-\sigma}=\bar{\Phi}^{-1}(\sigma) .
$$

Lemma 5 is proved.

For a function $f \in \mathcal{A}_{R}$ of the form (1) let $\nu(r, f)=\max \left\{n \in \mathbb{N}_{0}:\left|a_{n}\right| r^{n}=\mu(r, f)\right\}$ be its central index. It is well known that $\nu(r, f)=r(\ln \mu(r, f))_{+}^{\prime}$ for all $r \in(0, R)$. In addition, we have following result $([9])$.

Lemma 6. Let $R \in(0,+\infty],\left(n_{k}\right)$ be an increasing sequence of nonnegative integers, and $\left(c_{k}\right)$ be a sequence, increasing to $R$. If $\left(a_{n}\right)$ is a complex sequence such that $a_{n_{0}} \neq 0, a_{n}=0$ for each $n<n_{0}$ and

$$
\left|a_{n_{k+1}}\right|=\left|a_{n_{0}}\right| \prod_{j=0}^{k} c_{j}^{n_{j}-n_{j+1}}, \quad\left|a_{n}\right| \leq\left|a_{n_{k}}\right| c_{k}^{n_{k}-n}, \quad n \in\left(n_{k}, n_{k+1}\right)
$$

for all $k \geq 0$, then the power series (1) defines a function $f$, analytic in the disk $\mathbb{D}_{R}$ and such that for which: 
(i) $\nu(r, f)=n_{0}$ for all $r \in\left(0, c_{0}\right)$;

(ii) $\nu(r, f)=n_{k+1}$ for all $r \in\left[c_{k}, c_{k+1}\right)$ and $k \geq 0$.

Lemma 7. Let $\varepsilon>0$. Then there exists a number $\delta=\delta(\varepsilon)>0$ such that

$$
\left|1-\left(1+\frac{z}{n}\right)^{n}\right| \geq(1-\varepsilon)|z|
$$

for any $z \in \mathbb{D}_{\delta}$ and $n \in \mathbb{N}$.

Proof. Let $z \in \mathbb{C}, n \in \mathbb{N}$. Then

$$
\left|1-(1+z)^{n}\right| \geq 1+2 n|z|-(1+|z|)^{n} .
$$

Indeed, if $n \geq 2$, then, using the Newton binomial formula, we obtain

$$
\left|1-(1+z)^{n}\right|=\left|\sum_{k=1}^{n} C_{n}^{k} z^{k}\right| \geq n|z|-\sum_{k=2}^{n} C_{n}^{k}|z|^{k}=1+2 n|z|-(1+|z|)^{n} .
$$

If $n=1$, then inequality (13) turns to equality.

Further, since $e^{r}-1 \sim r, r \rightarrow 0$, then there exists $\delta>0$ such that $e^{r}-1 \leq(1+\varepsilon) r$, $r \in[0, \delta)$. Thus, if $z \in \mathbb{D}_{\delta}$ and $n \in \mathbb{N}$, then

$$
\left(1+\frac{|z|}{n}\right)^{n} \leq e^{|z|} \leq 1+(1+\varepsilon)|z|
$$

so, using inequality (13) with $z / n$ instead of $z$, we have

$$
\left|1-\left(1+\frac{z}{n}\right)^{n}\right| \geq 1+2|z|-\left(1+\frac{|z|}{n}\right)^{n} \geq 1+2|z|-(1+(1+\varepsilon)|z|)=(1-\varepsilon)|z| .
$$

Lemma 7 is proved.

\section{Proofs of theorems.}

Proof of Theorem 1. Let $f \in \mathcal{Z}_{R}$ be a function such that $T_{\Phi}(f) \leq 1$, and $C_{0}$ be the constant, defined by (6). Let us prove that for the function $f$ relation $(7)$ holds.

We set $\Psi(\sigma)=\ln M\left(e^{\sigma}, f\right)$ for each $\sigma<\ln R$. Then the condition $T_{\Phi}(f) \leq 1$ can be rewritten as $\Psi(\sigma) \leq(1+o(1)) \Phi(\sigma), \sigma \uparrow \ln R$. Since the function $\Psi$ is convex on the interval $(-\infty, \ln R)$, by Lemma 5 we obtain

$$
\Psi_{+}^{\prime}(\ln r) \leq(1+o(1)) \bar{\Phi}^{-1}(\ln r), \quad r \uparrow R .
$$

Suppose, on the contrary, that inequality (7) does not hold, that is, there exists a positive number $\lambda<C_{0}$ such that for the set $E$ of maximum modulus points $w$, satisfying the inequality

$$
d(w, f) \frac{\bar{\Phi}^{-1}(\ln |w|)}{|w|} \leq \lambda,
$$

we have $\sup E=R$. 
For each maximum modulus point $w$ of the function $f$ we fix the nearest zero of $f$ and denote this zero by $z(w)$. Note that by Lemma 1 for arbitrary $\rho \in(\max \{|w|,|z(w)|\}, R)$ the inequality

$$
M(|w|, f) \leq d(w, f) \frac{\rho M(\rho, f)}{\sqrt{\left(\rho^{2}-|w|^{2}\right)\left(\rho^{2}-|z(w)|^{2}\right)}}
$$

holds.

Since $\lambda<C_{0}$, for some $\mu>1$, according to (6), we have

$$
\lambda<\frac{\ln (2 \sqrt{\mu(\mu-1)})}{\mu} .
$$

Let $w \in E$. Put

$$
\rho(w)=|w|+\frac{\mu \lambda|w|}{\bar{\Phi}^{-1}(\ln |w|)} .
$$

Then $|w|<\rho(w)$. In addition, since

$$
|w-z(w)|=d(|w|, f) \leq \frac{\lambda|w|}{\Phi^{-1}(\ln |w|)},
$$

we have also $|z(w)|<\rho(w)$. It is also clear that $\rho(w) \sim|z(w)| \sim|w|$ as $|w| \uparrow R$.

Since

$$
\ln \rho(w)=\ln |w|+\ln \left(1+\frac{\mu \lambda}{\bar{\Phi}^{-1}(\ln |w|)}\right) \leq \ln |w|+\frac{\mu \lambda}{\bar{\Phi}^{-1}(\ln |w|)},
$$

by Lemma 4 we obtain $\bar{\Phi}^{-1}(\ln \rho(w)) \sim \bar{\Phi}^{-1}(\ln |w|)$ as $|w| \uparrow R$. Then, according to (14),

$$
\Psi_{+}^{\prime}(\ln \rho(w)) \leq(1+o(1)) \bar{\Phi}^{-1}(\ln |w|), \quad|w| \uparrow R .
$$

Using (17) and (18), we get

$$
\ln \frac{M(\rho(w), f)}{M(|w|, f)}=\int_{\ln |w|}^{\ln \rho(w)} \Psi_{+}^{\prime}(x) d x \leq \Psi_{+}^{\prime}(\ln \rho(w))(\ln \rho(w)-\ln |w|) \leq(1+o(1)) \mu \lambda
$$

as $|w| \uparrow R$. Therefore,

$$
\varliminf_{|w| \uparrow R} \frac{M(|w|, f)}{M(\rho(w), f)} \geq \frac{1}{e^{\mu \lambda}}
$$

Noting that the relations

$$
\rho^{2}(w)-|w|^{2} \sim \frac{2 \mu \lambda|w|^{2}}{\bar{\Phi}^{-1}(\ln |w|)}, \quad \rho^{2}(w)-|z(w)|^{2} \geq(1+o(1)) \frac{2(\mu-1) \lambda|w|^{2}}{\bar{\Phi}^{-1}(\ln |w|)},
$$

hold as $|w| \uparrow R$, and using inequality (15) with $\rho=\rho(w)$ and also (19), for $w \in E$ we obtain

$$
\begin{gathered}
\lambda \geq \varlimsup_{|w| \uparrow R} d(w, f) \frac{\bar{\Phi}^{-1}(\ln |w|)}{|w|} \geq \\
\geq \varlimsup_{|w| \uparrow R} \frac{M(|w|, f)}{M(\rho(w), f)} \frac{\sqrt{\left(\rho^{2}(w)-|w|^{2}\right)\left(\rho^{2}(w)-|z(w)|^{2}\right)}}{\rho(w)} \frac{\Phi^{-1}(\ln |w|)}{|w|} \geq \frac{2 \lambda \sqrt{\mu(\mu-1)}}{e^{\mu \lambda}},
\end{gathered}
$$

which contradicts (16). 
Proof of Theorem 4. Let $f \in \mathcal{Z}_{R}^{+}$be a function of the form (1), $\left(r_{j} e^{i \varphi_{j}}\right)$ be the sequence of all zeros of the function $f$, and $N:[0, R) \rightarrow(0,+\infty)$ be a function such that (10) holds. We prove that then the inequality (11) holds.

First of all, note that each of the numbers $r_{j} e^{-i \varphi_{j}}$ is also a zero of the function $f$ in view of the fact that its Taylor coefficients are nonnegative.

Suppose, on the contrary, that inequality (11) does not hold. Then, as stated above, there exists a number $\varepsilon \in(0, \pi)$ such that the set

$$
E=\left\{j \geq 0: 0 \leq \varphi_{j} \leq \frac{\pi-\varepsilon}{N\left(r_{j}\right)}\right\}
$$

is infinite.

Let $j \in E$. Then for every integer $k \in\left[0, N\left(r_{j}\right)\right]$ we have $0 \leq k \varphi_{j} \leq \pi-\varepsilon$, which implies that

$$
-\frac{\pi}{2}+\frac{\varepsilon}{2} \leq k \varphi_{j}-\left(\frac{\pi}{2}-\frac{\varepsilon}{2}\right) \leq \frac{\pi}{2}-\frac{\varepsilon}{2} .
$$

Taking

$$
\eta=\frac{\pi}{2}-\frac{\varepsilon}{2}, \quad \delta=\cos \left(\frac{\pi}{2}-\frac{\varepsilon}{2}\right),
$$

we see that $\cos \left(k \varphi_{j}-\eta\right) \geq \delta>0$ for all integers $k \in\left[0, N\left(r_{j}\right)\right]$. Therefore, in view of (10),

$$
\begin{gathered}
\left|\operatorname{Re}\left(e^{i \eta} f\left(r_{j} e^{i \varphi_{j}}\right)\right)\right|=\left|\sum_{k=0}^{\infty} a_{k} r_{j}^{k} \cos \left(k \varphi_{j}-\eta\right)\right| \geq \sum_{k \leq N\left(r_{j}\right)} a_{k} r_{j}^{k} \delta-\sum_{k>N\left(r_{j}\right)} a_{k} r_{j}^{k}= \\
=\delta M\left(r_{j}, f\right)-(1+\delta) \sum_{k>N\left(r_{j}\right)} a_{k} r_{j}^{k}=(\delta-o(1)) M\left(r_{j}, f\right), \quad j \rightarrow \infty .
\end{gathered}
$$

So, for all sufficiently large $j \in E$ we have $f\left(r_{j} e^{i \varphi_{j}}\right) \neq 0$. This contradicts the fact that the numbers $r_{j} e^{i \varphi_{j}}$ are zeros of the function $f$.

Proof of Theorem 2. Let $f \in \mathcal{Z}_{R}^{+}$be a function of the form $(1)$ such that $T_{\Phi}(f) \leq 1$. We prove that then inequality (8) holds.

Fix an arbitrary $\varepsilon>0$ and select $\delta>0$ so that the inequality

$$
\delta<1-\frac{1}{\sqrt{1+\varepsilon}}
$$

holds. Taking

$$
\eta=(1+\varepsilon)(1-\delta)^{2}-1,
$$

we see that $\eta>0$. Put $\Psi(\sigma)=\ln M\left(e^{\sigma}, f\right)$ for each $\sigma<\ln R$. From the condition $T_{\Phi}(f) \leq 1$ we have $\Psi(\sigma) \leq(1+\eta) \Phi(\sigma)$ for all $\sigma \in\left[\sigma_{1}, \ln R\right)$. Since the function $\Psi$ is convex on the interval $(-\infty, \ln R)$, by Lemma 5 we obtain

$$
\Psi_{+}^{\prime}(\ln r) \leq(1+\eta) \bar{\Phi}^{-1}(\ln r), \quad r \in\left[R_{1}, R\right) .
$$

Further, if $N>0, r \in(0, R)$, and $\rho \in(r, R)$, then

$$
\sum_{k>N} a_{n} r^{n}<\frac{M(r, f)}{e^{(\ln \rho-\ln r)\left(N-\Psi_{+}^{\prime}(\ln \rho)\right)}} .
$$


Indeed, since the coefficients $a_{n}$ are nonnegative, $M(r, f)=f(r)$ for all $r \in[0, R)$, and therefore

$$
\sum_{k>N} a_{n} r^{n}<\left(\frac{r}{\rho}\right)^{N} \sum_{k>N} a_{n} \rho^{n} \leq\left(\frac{r}{\rho}\right)^{N} M(\rho, f)=M(r, f)\left(\frac{r}{\rho}\right)^{N} e^{\Psi(\ln \rho)-\Psi(\ln r)} .
$$

It remains to take into account that

$$
\Psi(\ln \rho)-\Psi(\ln r)=\int_{\ln r}^{\ln \rho} \Psi_{+}^{\prime}(x) d x \leq(\ln \rho-\ln r) \Psi_{+}^{\prime}(\ln \rho) .
$$

As in Lemma 4, let $\sigma_{0}=\bar{\Phi}\left(x_{0}+0\right)$ and $y(\sigma)=\varphi\left(\bar{\Phi}^{-1}(\sigma)\right)$ for all $\sigma \in\left(\sigma_{0}, \ln R\right)$. Applying inequality $(21)$ with $N=(1+\varepsilon) \bar{\Phi}^{-1}(\ln r)$ and $\rho>r$ such that

$$
\ln \rho=\ln r+\frac{\delta \Phi(y(\ln r))}{\bar{\Phi}^{-1}(\ln r)},
$$

and using inequality (20) and Lemma 4, we obtain

$$
\begin{gathered}
\sum_{k>(1+\varepsilon) \bar{\Phi}^{-1}(\ln r)} a_{n} r^{n}<M(r, f) \exp \left(-\frac{\delta \Phi(y(\ln r))}{\bar{\Phi}^{-1}(\ln r)}\left((1+\varepsilon) \bar{\Phi}^{-1}(\ln r)-\frac{1+\eta}{1-\delta} \Psi_{+}^{\prime}(\ln r)\right)\right)= \\
=\frac{M(r, f)}{e^{\delta^{2}(1+\varepsilon) \Phi(y(\ln r))}}=o(M(r, f)), \quad r \uparrow R .
\end{gathered}
$$

Since $\varepsilon>0$ is arbitrary, hence by Theorem 4 we have

$$
\varliminf_{j \rightarrow \infty}\left|\varphi_{j}\right| \bar{\Phi}^{-1}\left(\ln r_{j}\right) \geq \pi
$$

where $\left(r_{j} e^{i \varphi_{j}}\right)$ is the sequence of all zeros of the function $f$.

Further, since the coefficients $a_{n}$ of the function $f$ are nonnegative, it is easy to prove (see, for example, [1]), that $d(w, f)=d(|w|, f)$ for an arbitrary maximum modulus point $w$ of this function. Therefore, if inequality (8) is not satisfied, then there exists a number $\lambda<\pi$ such that for the set

$$
E=\left\{r \in\left(e^{\sigma_{0}}, R\right): d(r, f) \frac{\bar{\Phi}^{-1}(\ln r)}{r} \leq \lambda\right\}
$$

we have $\sup E=R$.

For each point $r \in[0, R)$ we fix the number of the nearest zero of $f$ and denote this number by $j(r)$.

Let $r \in E$ and $r \uparrow R$. Then $d(r, f)=o(r)$, so it is clear from geometric considerations that $\varphi_{j(r)} \rightarrow 0$. Further, since $\left|r_{j(r)}-r\right| \leq d(r, f)$, we obtain $r_{j(r)} \sim r$. In addition,

$$
\ln r_{j(r)} \leq \ln (r+d(r, f)) \leq \ln \left(r+\frac{\lambda r}{\Phi^{-1}(\ln r)}\right) \leq \ln r+\frac{\lambda}{\Phi^{-1}(\ln r)},
$$

and therefore, by Lemma $4, \bar{\Phi}^{-1}\left(\ln r_{j(r)}\right) \leq(1+o(1)) \bar{\Phi}^{-1}(\ln r)$.

Using the above relations and the obvious inequality $r_{j(r)} \sin \left|\varphi_{j(r)}\right| \leq d(r, f)$ and assuming that $r \in E$, we get

$$
\varlimsup_{r \uparrow R}\left|\varphi_{j(r)}\right| \bar{\Phi}^{-1}\left(\ln r_{j(r)}\right)=\varlimsup_{r \uparrow R} \sin \left|\varphi_{j(r)}\right| \bar{\Phi}^{-1}\left(\ln r_{j(r)}\right) \leq \varlimsup_{r \uparrow R} d(r, f) \frac{\bar{\Phi}^{-1}(\ln r)}{r} \leq \lambda,
$$

which contradicts the inequality (22). 
Proof of Theorem 3. Let $\Phi \in \Omega_{\ln R}$. Suppose, as above, $x_{0}=\inf \{x>0: \Phi(\varphi(x))>0\}$. From condition (2) and the properties of the function $\widetilde{\Phi}$ given in Lemma 2 it follows that there exists an increasing sequence $\left(n_{k}\right)$ of nonnegative integers such that for it and for the sequences $\left(c_{k}\right)$ and $\left(r_{k}\right)$, where $c_{k}=\exp \left\{\bar{\Phi}\left(n_{k+1}\right)\right\}$ and $r_{k}=\exp \left\{\varphi\left(n_{k+1}\right)\right\}$ for all integers $k \geq 0$, we have $n_{0}=0, n_{1}>x_{0}$, and also

$$
\begin{gathered}
k n_{k} \ln r-\Phi(\ln r) \leq k n_{k} \ln c_{0}, \quad r \in\left[c_{k}, R\right), k \geq 0 \\
n_{k}=o\left(n_{k+1}\right), \quad k \rightarrow \infty \\
\min \left\{\left(n_{k+1}-n_{k}\right)\left(\ln c_{k+1}-\ln c_{k}\right),\left(n_{k+2}-n_{k+1}\right)\left(\ln c_{k+1}-\ln r_{k}\right)\right\} \geq 2 \ln (k+1), k \geq 0 .
\end{gathered}
$$

Put $a_{0}=1$,

$$
a_{n_{k+1}}=\prod_{j=0}^{k} c_{j}^{n_{j}-n_{j+1}}, \quad k \geq 0,
$$

and $a_{n}=0$ if $n \in\left(n_{k}, n_{k+1}\right)$ for some $k \geq 0$. By Lemma 6 the power series

$$
f(z)=\sum_{k=0}^{\infty} a_{n_{k}} z^{n_{k}}
$$

defines a function $f$, analytic in the disk $\mathbb{D}_{R}$, such that $\nu(r, f)=0$ for all $r \in\left(0, c_{0}\right)$ and $\nu(r, f)=n_{k+1}$ for all $r \in\left[c_{k}, c_{k+1}\right)$ and $k \geq 0$.

Let $r \in\left[c_{k}, c_{k+1}\right)$ and $k \geq 0$. Then

$$
\ln c_{k}=\bar{\Phi}\left(n_{k+1}\right)=\sup \left\{\sigma-\frac{\Phi(\sigma)}{n_{k+1}}: \sigma \in D_{\Phi}\right\} \geq \ln r-\frac{\Phi(\ln r)}{n_{k+1}} .
$$

From inequalities (26) and (23) we obtain, respectively,

$$
n_{k+1} \ln \frac{r}{c_{k}} \leq \Phi(\ln r), \quad n_{k} \ln \frac{c_{k}}{c_{0}} \leq n_{k} \ln \frac{r}{c_{0}} \leq \frac{\Phi(\ln r)}{k},
$$

so for every $k \geq 1$ we have

$$
\begin{gathered}
\ln \mu(r, f)=\ln \mu\left(c_{k}, f\right)+\int_{c_{k}}^{r} \frac{\nu(t, f)}{t} d t \leq n_{k} \ln \frac{c_{k}}{c_{0}}+n_{k+1} \ln \frac{r}{c_{k}} \leq \\
\leq \frac{\Phi(\ln r)}{k}+\Phi(\ln r)=\frac{k+1}{k} \Phi(\ln r) .
\end{gathered}
$$

This implies that $t_{\Phi}(f) \leq 1$.

We further note that equality (12), which follows from Lemma 2, can be rewritten as

$$
\bar{\Phi}(x)=\varphi(x)-\frac{\Phi(\varphi(x))}{x}, \quad x \in \mathbb{R} .
$$

Putting $x=n_{k+1}$, we get

$$
\ln c_{k}=\ln r_{k}-\frac{\Phi\left(\ln r_{k}\right)}{n_{k+1}}
$$

Since $\mu(r, f) \geq \mu(0, f)=1, r \in(0, R)$, we obtain, using (27),

$$
\ln \mu\left(r_{k}, f\right) \geq \int_{c_{k}}^{r_{k}} \frac{\nu(t, f)}{t} d t \geq n_{k+1} \ln \frac{r_{k}}{c_{k}}=\Phi\left(\ln r_{k}\right) .
$$


This and the inequality $t_{\Phi}(f) \leq 1$ imply that $t_{\Phi}(f)=1$.

Put

$$
\delta_{k}=\frac{1}{\sqrt{k+1}}, m_{k}=n_{k+1}-n_{k}, h_{k}=\frac{c_{k} \delta_{k}}{m_{k}}
$$

for all $k \geq 0$. Then, according to (24) and (27), for $k \geq k_{0}$ we obtain

$$
c_{k}+h_{k}=c_{k}\left(1+\frac{\delta_{k}}{m_{k}}\right) \leq c_{k}\left(1+\frac{\Phi\left(\ln r_{k}\right)}{n_{k+1}}\right) \leq c_{k} \exp \left\{\frac{\Phi\left(\ln r_{k}\right)}{n_{k+1}}\right\}=r_{k} .
$$

Note that

$$
\left(c_{k}-h_{k}\right)^{n_{k+1}} \sim c_{k}^{n_{k+1}} \sim\left(c_{k}+h_{k}\right)^{n_{k+1}}, \quad k \rightarrow \infty .
$$

Therefore, as $k \rightarrow \infty$, we have, uniformly for $r \in\left[c_{k}-h_{k}, c_{k}+h_{k}\right]$,

$$
a_{n_{k}} r^{n_{k}} \sim a_{n_{k}} c_{k}^{n_{k}}=\mu\left(c_{k}, f\right), \quad a_{n_{k+1}} r^{n_{k+1}} \sim a_{n_{k+1}} c_{k}^{n_{k+1}}=\mu\left(c_{k}, f\right) .
$$

For each integer $p \geq 0$ we set

$$
b_{p}=c_{p} e^{i \pi / m_{p}}, \quad C_{p}=\left\{z \in \mathbb{C}:\left|z-b_{p}\right|=h_{p}\right\}
$$

and consider the functions

$$
P_{p}(z)=a_{n_{p}} z^{n_{p}}+a_{n_{p+1}} z^{n_{p+1}}, \quad g_{p}(z)=f(z)-P_{p}(z) .
$$

Let $\delta$ be a number whose existence follows from Lemma 7 for $\varepsilon=1 / 2$, and let $z \in C_{p}$. Then $z=b_{p}+h_{p} e^{i \theta}$ for some $\theta \in \mathbb{R}$. For all $p \geq p_{0}$ we have $\delta_{p}<\delta$. Putting $|z|=r$ and using Lemmas 7 and (29), we get

$$
\begin{aligned}
\left|P_{p}(z)\right|= & \left|a_{n_{p}} z^{n_{p}}+a_{n_{p+1}} z^{n_{p+1}}\right|=a_{n_{p}} r^{n_{p}}\left|1+\left(\frac{z}{c_{p}}\right)^{m_{p}}\right|=a_{n_{p}} r^{n_{p}}\left|1+\left(e^{i \pi / m_{p}}+\frac{\delta_{p}}{m_{p}} e^{i \theta}\right)^{m_{p}}\right|= \\
& =a_{n_{p}} r^{n_{p}}\left|1-\left(1+\frac{\delta_{p}}{m_{p}} e^{i\left(-\pi / m_{p}+\theta\right)}\right)^{m_{p}}\right| \geq a_{n_{p}} r^{n_{p}} \frac{\delta_{p}}{2} \geq \frac{\delta_{p}}{3} \mu\left(c_{p}, f\right), \quad p \geq p_{1} .
\end{aligned}
$$

In addition, using (28) and (25), for every $p \geq p_{2}$ we have

$$
\begin{gathered}
\left|\sum_{k \leq p-1} a_{n_{k}} z^{n_{k}}\right| \leq \sum_{k \leq p-1} a_{n_{k}}\left(c_{p}+h_{p}\right)^{n_{k}}=a_{n_{p}}\left(c_{p}+h_{p}\right)^{n_{p}} \sum_{k \leq p-1} \frac{a_{n_{k}}}{a_{n_{p}}}\left(c_{p}+h_{p}\right)^{n_{k}-n_{p}} \leq \\
\leq 2 \mu\left(c_{p}, f\right) \sum_{k \leq p-1} \frac{a_{n_{k}}}{a_{n_{p}}} c_{p}^{n_{k}-n_{p}}=2 \mu\left(c_{p}, f\right) \sum_{k \leq p-1} \prod_{j=k}^{p-1}\left(\frac{c_{j}}{c_{p}}\right)^{n_{j+1}-n_{j}} \leq \\
\leq 2 \mu\left(c_{p}, f\right) \sum_{k \leq p-1}\left(\frac{c_{p-1}}{c_{p}}\right)^{n_{p}-n_{p-1}}=2 \mu\left(c_{p}, f\right) p\left(\frac{c_{p-1}}{c_{p}}\right)^{n_{p}-n_{p-1}} \leq \frac{2}{p} \mu\left(c_{p}, f\right) ; \\
\left|\sum_{k \geq p+2} a_{n_{k}} z^{n_{k}}\right| \leq \sum_{k \geq p+2} a_{n_{k}}\left(c_{p}+h_{p}\right)^{n_{k}}=a_{n_{p+1}}\left(c_{p}+h_{p}\right)^{n_{p+1}} \sum_{k \geq p+2} \frac{a_{n_{k}}}{a_{n_{p+1}}}\left(c_{p}+h_{p}\right)^{n_{k}-n_{p+1}} \leq \\
\leq 2 \mu\left(c_{p}, f\right) \sum_{k \geq p+2} \frac{a_{n_{k}}}{a_{n_{p+1}}} r_{p}^{n_{k}-n_{p+1}}=2 \mu\left(c_{p}, f\right) \sum_{k \geq p+2} \prod_{j=p+1}^{k-1}\left(\frac{r_{p}}{c_{j}}\right)^{n_{j+1}-n_{j}} \leq
\end{gathered}
$$




$$
\begin{gathered}
\leq 2 \mu\left(c_{p}, f\right) \sum_{k \geq p+2}\left(\frac{r_{p}}{c_{k-1}}\right)^{n_{k}-n_{k-1}} \leq 2 \mu\left(c_{p}, f\right) \sum_{k \geq p+2}\left(\frac{r_{k-2}}{c_{k-1}}\right)^{n_{k}-n_{k-1}} \leq \\
\leq 2 \mu\left(c_{p}, f\right) \sum_{k \geq p+2} \frac{1}{(k-1)^{2}} \leq \frac{2}{p} \mu\left(c_{p}, f\right) .
\end{gathered}
$$

So for all $p \geq p_{3}$ and $z \in C_{p}$ we get

$$
\left|g_{p}(z)\right| \leq \frac{4}{p} \mu\left(c_{p}, f\right)<\frac{\delta_{p}}{3} \mu\left(c_{p}, f\right) \leq\left|P_{p}(z)\right| .
$$

Thus, according to Rouché's theorem, the function $f$ has in the disk $\left\{z \in \mathbb{C}:\left|z-b_{p}\right|<h_{p}\right\}$ the same number of zeros as the binomial $P_{p}$. Since $b_{p}$ is a zero of $P_{p}$, in this disk the function $f$ has a zero, which we denote by $z_{p}$. If $p \rightarrow \infty$, then

$$
\left|b_{p}-z_{p}\right|<h_{p}=\delta_{p} c_{p} / m_{p}=o\left(c_{p} / n_{p+1}\right), \quad\left|c_{p}-b_{p}\right|=\left|c_{p}-c_{p} e^{i \pi / m_{p}}\right| \sim c_{p} \pi / n_{p+1} .
$$

This implies, by the triangle inequality, that

$$
\left|c_{p}-z_{p}\right| \sim c_{p} \pi / n_{p+1}=c_{p} \pi / \bar{\Phi}^{-1}\left(\ln c_{p}\right), \quad p \rightarrow \infty
$$

Therefore,

$$
\varliminf_{r \uparrow R} d(r, f) \frac{\bar{\Phi}^{-1}(\ln r)}{r} \leq \varliminf_{p \rightarrow \infty} d\left(c_{p}, f\right) \frac{\bar{\Phi}^{-1}\left(\ln c_{p}\right)}{c_{p}} \leq \varliminf_{p \rightarrow \infty}\left|c_{p}-z_{p}\right| \frac{\bar{\Phi}^{-1}\left(\ln c_{p}\right)}{c_{p}}=\pi .
$$

This and Theorem 2 imply equality (9).

Next, we note that the above estimates imply the relation $M\left(c_{k}, f\right) \sim 2 \mu\left(c_{k}, f\right), k \rightarrow \infty$. Since the functions $\ln M(r, f)$ and $\ln \mu(r, f)$ are convex and linear with respect to $\ln r$ on $\left[c_{k}, c_{k+1}\right]$, respectively, and $\mu(r, f) \leq M(r, f)$ by the Cauchy inequality, we have

$$
0 \leq \ln M(r, f)-\ln \mu(r, f) \leq \ln 3
$$

for all $r<R$ sufficiently close to $R$. This and the equality $t_{\Phi}(f)=1$ imply that $T_{\Phi}(f)=1$.

\section{REFERENCES}

1. I.V. Ostrovskii, Distance between a maximum modulus point of an entire function and its zero set, Operator theory, subharmonic functions, Kyiv: Naukova Dumka, 1991, 67-75. (in Russian)

2. I. Ostrovskii, A.E. Üreyen, Distance between a maximum modulus point and zero set of an entire function, Complex Variables, Theory Appl., 48 (2003), №7, 583-598.

3. I. Ostrovskii, A.E. Üreyen, Maximum modulus points and zero sets of entire functions of regular growth, C. R. Acad. Sci. Paris. Ser. I., 341 (2005), №8, 481-484.

4. I. Ostrovskii, A.E. Üreyen, On maximum modulus points and zero sets of entire functions of regular growth, Rocky Mt. J. Math., 38 (2008), №2, 583-618.

5. A.E. Üreyen, On maximum modulus points and the zero set for an entire function of either zero or infinite order, Comput. Methods Funct. Theory, 4 (2005), №2, 341-354.

6. S.I. Fedynyak, On maximum modulus points and zero set for an entire function, Mat. Stud., 30 (2008), №2, 169-172. 
7. M.A. Evgrafov, Asymptotic estimates and entire functions, Moscow: Nauka, 1979. (in Russian)

8. T.Ya. Hlova, P.V. Filevych, Generalized types of the growth of Dirichlet series, Carpathian Math. Publ., 7 (2015), №2, 172-187.

9. P.V. Filevych, On the slow growth of power series convergent in the unit disk, Mat. Stud., 16 (2001), №2, $217-221$.

Department of Applied Mathematics and Statistics Ukrainian Catholic University, Lviv, Ukraine napets.fed@gmail.com Department of Computational Mathematics and Programming Lviv Polytechnic National University, Lviv, Ukraine p.v.filevych@gmail.com 